\title{
EQUIVALENT CONDITIONS FOR DECOMPOSABLE OPERATORS
}

\author{
RIDGLEY LANGE
}

\begin{abstract}
Several new characterizations of an arbitrary decomposable operator on Banach space are given; for example, one of these is in terms of spectral conditions on an arbitrary invariant subspace, while another uses the spectral manifold $X_{T}\left(G^{-}\right)$(rather than $X_{T}(F)$ ). From these results a short proof of Frunză's duality theorem is derived. Finally we give sufficient conditions that the predual of a decomposable operator is of the same class.
\end{abstract}

1. Introduction. In this paper we present several new characterizations of a decomposable operator in the sense of Foiaş (see \$2, Theorem 1). Condition (3) below gives an equivalence in terms of an arbitrary invariant subspace of the given operator satisfying certain spectral properties. Another condition (5) characterizes a decomposable operator among the quasidecomposable operators. A final equivalent condition (6) is similar to one given in [6], but it is stated for open rather than closed sets.

Under the restriction that the spectrum of a given operator is nowhere dense, the operator is decomposable if and only if its adjoint is decomposable. This is proved in $\$ 3$.

Throughout the rest of the paper $T$ will denote a bounded linear operator on the complex Banach space $X$. A spectral maximal space (for $T$ ) is a closed invariant linear manifold $M$ (subspace) which contains every invariant subspace $N$ of $T$ for which $\sigma(T \mid N) \subset \sigma(T \mid M)$. Let $C$ be the complex plane and let $\left\{G_{1}, G_{2}, \ldots, G_{n}\right\}$ be a finite open cover of $C$. Then $T$ is said to be decomposable if there are spectral maximal spaces $M_{1}, M_{2}, \ldots, M_{n}$ such that $X=M_{1}+\cdots+M_{n}$ and $\sigma\left(T \mid M_{i}\right) \subset$ $G_{i}$ (equivalently, $\sigma\left(T \mid M_{i}\right) \subset G_{i}^{-}$) for $i=1,2, \ldots, n$. If $n<2, T$ is called 2-decomposable.

The elementary properties of a decomposable operator $T$ may be found in [4] and can be summarized as follows. It has the single-valued extension property (SVEP), which implies that for each set $F \subset C$ the spectral manifold $X_{T}(F)=$ $\{x \in X:(\lambda-T) f(\lambda)=x$ for some analytic $f: C \backslash F \rightarrow X\}$ is defined and invariant under $T$. If $F$ is closed, then $X_{T}(F)$ is norm-closed and hence spectral maximal such that $\sigma\left(T \mid X_{T}(F)\right) \subset F$; moreover, every spectral maximal space has this form.

All of the above remarks hold for a 2-decomposable operator, and it is clear that $T$ is 2-decomposable if it has the SVEP and $X=X_{T}\left(G_{1}^{-}\right)+X_{T}\left(G_{2}^{-}\right)$for every pair of open sets $G_{1}, G_{2}$ covering $C$.

Received by the editors November 10, 1978 and, in revised form, June 21, 1979. AMS (MOS) subject classifications (1970). Primary 47B99; Secondary 47A15. Key words and phrases. Decomposable operator, spectral maximal space. 
Let $X^{\prime}$ be the dual space of $X$, and let $A \subset X, B \subset X^{\prime}$. Then $A^{\perp}$ [resp. $B^{\perp}$ ] denotes the annihilator of $A$ in $X^{\prime}$ [resp. annihilator of $B$ in $X$ ]. We write $M^{-}$for the norm-closure of $M \subset X$ and $F^{\circ}$ for the interior of $F \subset C$. Let $M$ be a $T$-invariant subspace. Then $T^{M}$ will denote the operator induced on $X / M$ by $T$. For $M=X_{T}(F)$, we write $T^{F}$ for the induced operator, and $T^{G}$ if $M=X_{T}(G)^{-}$.

Finally, let us recall that $T$ is quasidecomposable if it has the SVEP and the manifolds $X_{T}\left(G_{i}^{-}\right)$are closed and span $X$ for each cover $\left\{G_{i}\right\}$ of $C$ (i= $1,2, \ldots, n)$.

\section{The main result.}

THEOREM 1. Let $T$ be a bounded linear operator on the Banach space $X$, and let $T^{\prime}$ be its adjoint on the dual space $X^{\prime}$. Then the following assertions are equivalent.

(1) $T$ is decomposable.

(2) $T$ is 2-decomposable.

(3) For every open set $G$ in $C$ there is a $T$-invariant subspace $Y$ such that $\sigma(T \mid Y) \subset G^{-}$and $\sigma\left(T^{Y}\right) \subset C \backslash G$.

(4) $T$ has the SVEP and for $F$ closed $X_{T}(F)$ is closed such that $\sigma\left(T^{F}\right) \subset C \backslash F^{\circ}$.

(5) $T$ is quasidecomposable and for $F$ closed $\sigma\left(T^{\prime} \mid X_{T}(C \backslash F)^{\perp}\right) \subset F$.

(6) $T$ has the SVEP and for $G$ open $X_{T}\left(G^{-}\right)$is closed and $\sigma\left(T^{G}\right) \subset C \backslash G$.

The equivalence (1) $\Leftrightarrow$ (2) was proved by Radjabalipour [9], and (2) $\Leftrightarrow$ (4) was proved in [6]. The characterizations (3), (5) and (6) are new. Our proof of the theorem will use the following lemmas.

LEMMA 1 [7, p. 221]. Let $M$ and $N$ be closed linear manifolds in $X$. Then $M+N$ is closed iff $M^{\perp}+N^{\perp}$ is closed and in this case $(M \cap N)^{\perp}=M^{\perp}+N^{\perp}$.

LEMMA 2 [1, p. 434]. Suppose $T$ has the SVEP and $F_{1}$ and $F_{2}$ are disjoint closed sets. If $X_{T}\left(F_{1} \cup F_{2}\right)$ is closed, then $X_{T}\left(F_{i}\right)(i=1,2)$ are closed and $X_{T}\left(F_{1} \cup F_{2}\right)=$ $X_{T}\left(F_{1}\right) \oplus X_{T}\left(F_{2}\right)$.

The next lemma follows from Liouville's theorem.

LEMMA 3. Suppose $T$ and its adjoint $T^{\prime}$ both have the SVEP. If $F$ and $H$ are disjoint sets in $C$, then $X_{T}(F)^{\perp} \supset X_{T^{\prime}}^{\prime}(H)$ and $X_{T}(F) \subset X_{T^{\prime}}^{\prime}(H)^{\perp}$.

LEMMA 4 [3]. If $T$ has the SVEP and is surjective on $X$, then $T$ is injective.

To state the final lemma required for the proof of Theorem 1, we recall the notion of an analytically invariant subspace (see $[8, \S 2]$ for details). The subspace $M$ in $X$ is analytically invariant for $T$ if for each analytic function $f: D \rightarrow X$ the relation $(\lambda-T) f(\lambda) \in M$ for $\lambda \in D$ implies that $f(\lambda) \in M$ for $\lambda \in D$. An easy argument shows that every analytically invariant subspace is invariant.

LEMMA 5. Let $T$ have the SVEP. If $Y$ is a $T$-invariant subspace such that $\sigma(T \mid Y) \cap \sigma\left(T^{Y}\right)$ is nowhere dense in $C$, then $Y$ is analytically invariant. 
Proof. Let $f: D \rightarrow X$ be an analytic function such that $D$ is connected and $(\lambda-T) f(\lambda) \in Y$ for $\lambda \in D$. Suppose $E=D \cap \rho(T \mid Y) \neq \varnothing$ and let $h(\lambda)=$ $(\lambda-T) f(\lambda)$ and $g(\lambda)=R(\lambda ; T \mid Y) h(\lambda)$ for $\lambda \in E$. It follows that $(\lambda-T) g(\lambda)=$ $(\lambda-T) f(\lambda)$ for $\lambda \in E$. Since $T$ has the SVEP, $f(\lambda)=g(\lambda)$ for $\lambda \in E$, so $f(\lambda) \in Y$ for all $\lambda \in D$ by analytic continuation. On the other hand, $D \subset \sigma(T \mid Y)$ implies that $G=D \cap \rho\left(T^{Y}\right) \neq \varnothing$. On $G$ we obtain $\left(\lambda-T^{Y}\right) \hat{f}(\lambda)=0$ where $\hat{f}(\lambda)$ denotes the coset $f(\lambda)+Y$. Hence $\hat{f}=0$ on $G$, so $f(\lambda) \in Y$ for all $\lambda \in D$.

Proof OF THEOREM 1. Our argument will consist of proving the implications (2) $\Rightarrow(4) \Rightarrow(3) \Rightarrow$ (2) and (4) $\Rightarrow$ (5) $\Rightarrow$ (6) $\Rightarrow$ (3).

(2) $\Rightarrow$ (4). Let $\lambda \in F^{\circ}$. Without loss of generality suppose $\lambda=0$. Let $H$ be an open set such that $H$ and $F^{\circ}$ cover $C$ and $0 \notin H^{-}$, and let $M$ and $N$ be spectral maximal spaces such that $X=M+N, \sigma(T \mid M) \subset F^{\circ}$ and $\sigma(T \mid N) \subset H$. For $x$

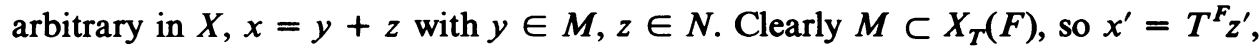
where $x^{\prime}$ denotes the coset $x+X_{T}(F)$; hence $T^{F}$ is surjective. Now suppose that $T^{F} x^{\prime}=0$ for some $x \in X$. Since $T x \in X_{T}(F)$ we can prove that $T^{F}$ is injective if we show $x \in X_{T}(F)$. Let $V$ be the span of $x$ and $X_{T}(F)$. Hence $V$ is an invariant subspace under $T$. It suffices to show that $V \subset X_{T}(F)$ and this will follow from $\sigma(T \mid V) \subset F$ since $X_{T}(F)$ is spectral maximal. Let $\mu \notin F$. Then $\mu \neq 0$, so the equalities $\mu x=(\mu-T) x+T x=(\mu-T)(x+y)$ for some $y \in X_{T}(F)$ show that $x$ has a preimage in $V$ under $\mu-T$. It follows that $\mu-T$ is surjective on $V$ and hence bijective on $V$ by Lemma 4. Thus $x \in X_{T}(F)$ and $T^{F}$ is bijective. Hence (4) is proved.

(4) $\Rightarrow(3)$. Let $G$ be open and put $F=G^{-}$. By (4), $X_{T}(F)$ is closed; hence $\sigma\left(T \mid X_{T}(F)\right) \subset F$. Moreover, $T^{F}$ has spectrum contained in $C \backslash G$. Thus, (3) follows if we put $Y=X_{T}\left(G^{-}\right)$.

(3) $\Rightarrow(2)$. Let $\left\{G_{1}, G_{2}\right\}$ be any open cover of $C$. By [8, Theorem 3.1], we need only prove that there exist analytically invariant subspaces $Y_{1}, Y_{2}$ such that $X=Y_{1}+Y_{2}$ and $\sigma\left(T \mid Y_{i}\right) \subset G_{i}^{-}$. To see this put $G=G_{1} \cap G_{2}$ and let $Y$ be as in (3) corresponding to $G$. By Lemma 5 and (3), $Y$ is analytically invariant to $T$, and $\sigma\left(T^{Y}\right) \subset C \backslash G_{1} \cap G_{2}=\left(C \backslash G_{1}\right) \cup\left(C \backslash G_{2}\right)$. Since the last union is a disjoint one of closed sets, we can by the Riesz-Dunford functional calculus write the quotient space $X / Y$ as a direct sum $W_{1} \oplus W_{2}$ of $T^{Y}$-invariant subspaces such that $\sigma\left(T^{Y} \mid W_{i}\right) \subset C \backslash G_{i}(i=1,2)$. Let $\phi: X \rightarrow X / Y$ be the natural surjection, and put $Y_{i}=\phi^{-1}\left(W_{j}\right), \quad j \neq i(i, j=1,2)$. Then $Y_{i}$ is $T$-invariant, $X=Y_{1}+Y_{2}$ and $\sigma\left(T \mid Y_{i}\right) \subset \sigma\left(T^{Y} \mid W_{j}\right) \cup \sigma(T \mid Y) \subset G_{i}^{-} \cap G^{-} \subset \bar{G}_{i}$. It remains to prove that $Y_{i}$ is analytically invariant to $T$. Let $f: D \rightarrow X$ be analytic on the connected domain $D$ such that $(\lambda-T) f(\lambda) \in Y_{1}$. For $\mu \in D$ fixed there is an open disc $E$ in $D$ with center $\mu$ and analytic functions $f_{i}: E \rightarrow Y_{i}(i=1,2)$ such that $f=f_{1}+f_{2}$ on $E$ (see [2, proof of Theorem 8]). In the quotient space $X / Y$,

$$
\left(\lambda-T^{Y}\right) \hat{f}_{2}(\lambda)=\left(\Lambda-T^{Y}\right)\left[\hat{f}(\lambda)-\hat{f}_{1}(\lambda)\right] \in \phi\left(Y_{1}\right) \cap \phi\left(Y_{2}\right)=(0),
$$

where $\hat{f}=\phi \circ f$. Thus by the analytic invariance of $Y, f_{2}(\lambda) \in Y$ for $\lambda \in E$; and it follows that $f(\lambda) \in Y_{1}$ for all $\lambda \in D$. This proves that $Y_{1}$ (and hence $Y_{2}$ also) is analytically invariant, and (3) is proved. 
(4) $\Rightarrow(5)$. Since (4) $\Leftrightarrow(1)$, it is obvious that $T$ is quasidecomposable; hence it suffices to prove that $\sigma\left(T^{\prime} \mid X_{T}(C \backslash F)^{\perp}\right) \subset F$. Let $G=C \backslash F$. Let $\lambda \in G$ and suppose that $\lambda=0$. Let $\left\{F_{n}\right\}$ be an increasing sequence of closed sets whose union is $G$ such that $0 \in F_{1}^{\circ}$. Then $X_{T}(G)=X_{T}\left(\cup F_{n}\right)=\cup X_{T}\left(F_{n}\right)$, and so $X_{T}(G)^{\perp}=$ $\cap X_{T}\left(F_{n}\right)^{\perp}$. Let $W=X_{T}(G)^{\perp}$ and $W_{n}=X_{T}\left(F_{n}\right)^{\perp}$. Then $W_{n}$ and $W$ are $T^{\prime}$ invariant and $T^{\prime} \mid W_{n}$ can be identified with the adjoint of the corresponding operator induced by $T$ on $X / X_{T}\left(F_{n}\right)$. By (4), $T^{\prime}$ is bijective on each $W_{n}$, and it follows that $T^{\prime}$ is bijective on $W$. Hence the desired inclusion is proved.

(5) $\Rightarrow(6)$. By definition $T$ has the SVEP and $X_{T}\left(G^{-}\right)$is closed for all open $G$. Since $T^{\prime} \mid X_{T}(G)^{\perp}$ is the adjoint of $T^{G},(6)$ follows from the inclusion in (5).

$(6) \Rightarrow(3)$. Let $G$ be open and let $Y=X_{T}(G)^{-}$. Since $X_{T}\left(G^{-}\right)$is closed by hypothesis, $\sigma(T \mid Y) \subset G^{-}$and the inclusion $\sigma\left(T^{G}\right) \subset C \backslash G$ proves (3). This completes the proof of the theorem.

The theorem allows us to prove a duality result of Frunză [5], which we shall use later.

COROLlaRY 1. Let $T$ be 2-decomposable. Then the adjoint $T^{\prime}$ is also 2-decomposable and $X_{T^{\prime}}^{\prime}(F)=X_{T}(C \backslash F)^{\perp}$ for all closed $F$.

Proof. Let $\left\{G_{1}, G_{2}\right\}$ be a cover of $C$ and let $H_{i}=C \backslash G_{i}^{-}$. Then the $H_{i}^{-}$are closed and disjoint; hence Lemma 2 implies that $X_{T}\left(H_{1}^{-}\right)+X_{T}\left(H_{2}^{-}\right)$is direct and thus closed. It follows that $X_{T}\left(H_{1}\right)^{-}+X_{T}\left(H_{2}\right)^{-}$is closed in $X$; hence, by Lemma 1, $X_{T}\left(H_{1}\right)^{\perp}+X_{T}\left(H_{2}\right)^{\perp}$ is closed in $X^{\prime}$ and because the $H_{i}^{-}$are disjoint $X^{\prime}=$ $\left[X_{T}\left(H_{1}\right)^{-} \cap X_{T}\left(H_{2}\right)^{-}\right]^{\perp}=X_{T}\left(H_{1}\right)^{\perp}+X_{T}\left(H_{2}\right)^{\perp}$. Put $W_{i}=X_{T}\left(H_{i}\right)^{\perp}$. Then $\sigma\left(T^{\prime} \mid W_{i}\right) \subset C \backslash H_{i}=G_{i}^{-}$(by (5) above). It follows from [2, Theorem 8] that $T^{\prime}$ has the SVEP. Thus $W_{i} \subset X_{T^{\prime}}^{\prime}\left(G_{i}^{-}\right)$and the reverse inclusion results from Lemma 3. Since $X_{T^{\prime}}^{\prime}\left(G_{i}{ }^{-}\right)$are closed and have linear sum $X^{\prime}, T^{\prime}$ is 2-decomposable. The identity $X_{T^{\prime}}^{\prime}(F)=X_{T}(C \backslash F)^{\perp}$ follows from (5) and Lemma 3.

3. Preduals. In this section we seek sufficient conditions under which the implication in Corollary 1 is reversible. To do this we need the following lemma. By Corollary 1 each $X_{T^{\prime}}^{\prime}(F)$ is weak*-closed. The next lemma (whose proof was corrected by the referee) may be considered a generalization of this fact.

LEMMA 6. Let $S$ be the adjoint operator on some dual space $X^{\prime}$. If $S$ has the SVEP, then every spectral maximal space of $S$ is weak*-closed.

Proof. Let $M$ be a spectral maximal space of $S$, and let $N$ be the linear hull of the weak*-closure of the unit ball of $M$. Hence $N$ is a norm closed $S$-invariant subspace of $X^{\prime}$. Fix $u \in N$. Then $u$ is the weak*-limit of a uniformly bounded net $\left\{u_{\alpha}\right\}$ in $M$. For $\lambda \notin \sigma(S \mid M)$ there is a uniformly bounded net $\left\{v_{\alpha}\right\}$ in $X^{\prime}$ such that $(\lambda-S) v_{\alpha}=u_{\alpha}$. Now there is $v \in X^{\prime}$ every weak*-neighborhood of which contains a cofinal subnet of $\left\{v_{\alpha}\right\}$. It follows from the weak*-continuity of $S$ that $(\lambda-S) v$ $=u$, and moreover, $v \in N$. Hence $\lambda-S$ is surjective on $N$. Because $S \mid N$ also has the SVEP, by Lemma $4, \lambda-S$ is bijective on $N$ and thus $\sigma(S \mid N) \subset \sigma(S \mid M)$ and we have $N=M$. By the Krein-Šmulian theorem $M$ is weak*-closed. 
THeORem 2. Let $T$ be an operator on $X$ with adjoint $T^{\prime}$ on $X^{\prime}$. Let $S$ be the adjoint of $T^{\prime}$, and suppose that $X$ (considered as subspace of $X^{\prime \prime}$ ) is analytically invariant under $S$. Then $T$ is decomposable if $T^{\prime}$ is decomposable. In particular, if $\sigma(T)$ is nowhere dense, then $T^{\prime}$ is decomposable if and only if $T$ is.

Proof. By (1) $\Leftrightarrow(2)$ of Theorem 1 we may prove the present result in terms of 2-decomposability. Let $T^{\prime}$ be 2-decomposable and let $\left\{G_{1}, G_{2}\right\}$ be an open cover of $C$. Let $W_{i}=X_{T^{\prime}}^{\prime}\left(C \backslash G_{i}^{-}\right)^{-}$and put $M_{i}=W_{i}^{\perp}(i=1,2)$. Then $M_{i}^{\perp}$ is the weak*closure of $W_{i}$ in $X^{\prime}$. Letting $F_{i}=C \backslash G_{i}$, we see that the $F_{i}$ are closed disjoint sets; hence the inclusion $\sigma\left(T^{\prime} \mid W_{i}\right) \subset F_{i}$ implies that $M_{1}{ }^{\perp}+M_{2}^{\perp}$ is closed by Lemmas 2 and 6. Hence $M_{1}+M_{2}$ is closed by Lemma 1 and thus $X=M_{1}+M_{2}$ follows from the relation $\left(M_{1}+M_{2}\right)^{\perp}=M_{1}^{\perp} \cap M_{2}^{\perp}=(0)$. Since $S$ is decomposable by Corollary 1, it has the SVEP and so the restriction $T=S \mid X$ has this property also. Thus the linear manifolds $X_{T}\left(G_{i}^{-}\right)$are defined.

We complete the proof of the first conclusion of the theorem by showing that $M_{i}=X_{T}\left(G_{i}^{-}\right)$, i.e. $X_{T}\left(G_{i}^{-}\right)$are closed and their linear sum is $X$. By Lemma 3 we have $X_{T}\left(G_{i}^{-}\right) \subset W_{i}^{\perp}=M_{i}$. For the reverse inclusion, note that, by the last statement of Corollary $1, X_{S}^{\prime \prime}\left(G_{i}^{-}\right)=W_{i}^{a}=$ the annihilator of $W_{i}$ in $X^{\prime \prime}$ (the dual of $\left.X^{\prime}\right)$ and, by Corollary 1, (4) of Theorem 1 and Lemma $5, X_{S}^{\prime \prime}\left(G_{i}{ }^{-}\right)$is analytically invariant under $S$. Now let $V_{i}=X \cap X_{S}^{\prime \prime}\left(G_{i}^{-}\right)$where $X$ is considered a subspace of $X^{\prime \prime}$. Intersections of analytically invariant subspaces are obviously analytically invariant; hence by hypothesis and the previous remark $V_{i}$ is analytically invariant under $S$. By [8, Proposition 1.13(1), p. 231], $V_{i}$ is analytically invariant under $S \mid X_{S}^{\prime \prime}\left(G_{i}^{-}\right)$; hence by [8, Corollary 1.4, p. 227], $\sigma\left(S \mid V_{i}\right) \subset \sigma\left(S \mid X_{S}^{\prime \prime}\left(G_{i}^{-}\right)\right) \subset G_{i}^{-}$. Regarding $V_{i}$ now as $T$-invariant, we see that $M_{i} \subset V_{i} \subset X_{T}\left(G_{i}{ }^{-}\right)$and we infer that $X_{T}\left(G_{i}^{-}\right)$are closed. Hence $T$ is 2-decomposable.

To prove the final assertion of the theorem, merely note that if $\sigma(T)$ is nowhere dense, then by Lemma 5 and by [8, Proposition 1.3, p. 227], $X$ is analytically invariant under $S$. Now apply the first part of the theorem and Corollary 1 .

REMARK. The hypotheses of Theorem 2 make the following question natural: Is the Banach space $X$ analytically invariant under the second adjoint $T^{\prime \prime}$ of an operator $T$ (on $X$ ), at least if $T^{\prime}$ is decomposable? A positive answer would, of course, give the general result that the predual of any decomposable operator is decomposable.

Added September, 1980. Since this paper was written, the author has learned that Ş. Frunză has proved Theorem 2 in general, i.e. the predual of a decomposable operator is decomposable. It might also be added that the implication $(3) \Rightarrow(2)$ in the proof of Theorem 1 may be shortened by using the fact that $T$ has the spectral decomposition property [2], and that such operators are decomposable (see E. Albrecht, On decomposable operators, Integral Equations Operator Theory 2 (1979), $1-10)$.

\section{REFERENCES}

1. C. Apostol, Roots of decomposable operator-valued functions, Rev. Roumaine Math. Pures Appl. 13 (1968), 433-438.

2. I. Erdélyi and R. Lange, Operators with spectral decomposition properties, J. Math. Anal. Appl. 66 (1978), 1-19. 
3. J. Finch, The single-valued extension property in Banach spaces, Pacific J. Math. 48 (1975), 61-69.

4. C. Foiaş, Spectral maximal spaces and decompasable operators, Arch. Math. (Basel) 14 (1963), 341-349.

5. Ş. Frunză, A duality theorem for decomposable operators, Rev. Roumaine Math. Pures Appl. 16 (1971), 1055-1058.

6. A. Jafarian and F.-H. Vasilescu, A characterization of 2-decomposable operators, Rev. Roumaine Math. Pures Appl. 19 (1974), 769-771.

7. T. Kato, Perturbation theory for linear operators, Springer-Verlag, Heidelberg, 1966.

8. R. Lange, Analytically decomposable operators, Trans. Amer. Math. Soc. 244 (1978), 225-240.

9. M. Radjabalipour, On equivalence of decomposable and 2-decomposable operators, Pacific J. Math. 28 (1978), 243-247.

Department of Mathematics, Youngstown State University, Youngstown, OhIo 44555 